\title{
Therapeutic Role of Oral Water Soluble Contrast in Treatment of Early Adhesive Intestinal Obstruction in Pediatric Population
}

\author{
Mohamed Yousef Batikhe, ${ }^{1}$ Nezar A. Abo Halawa ${ }^{2}$ \\ ${ }^{1}$ Pediatric Surgery Unit, Department of General Surgery, Sohag University, Egypt \\ 2Pediatric Surgery Unit, Department of General Surgery, South Valley University, Egypt
}

Introduction and objectives: Adhesive bowel obstruction is considered one of the commonest causes of intestinal obstruction managed in daily practice, medical schools differ in management plans from conservative to interventional measures with increased morbidity and mortality with recurrent surgical interventions, the aim of our study is to evaluate the therapeutic role of oral water soluble contrast (urographin) in treatment of early adhesive intestinal obstruction.

Patients and methods: All pediatric patients presented at our units in the period from December 2016 to December 2017 by early adhesive intestinal obstruction were included in this study. All patients were given conservative treatment for 24 hours (nothing per mouth, insertion of nasogastric tube, and intravenous fluids). Patients with failure of improvement after 24 hours were divided to 2 groups in the first group we continued the same conservative measures for 48 hours, in the second group we used single oral dose of urographin. Follow up of the patients was done clinically and radiologically for another 48 hours. Demographic data, type of first surgery, results, complications, and outcome all were collected and statistically analyzed.

Results: The study included 35 patients, oral urographin was used in 14 patients, and we continued traditional measures in 21 patients. The mean duration of hospital stay in the urographin group was $3.36 \pm 0.49$ while in the conservative group was $6.24 \pm 0.89$ with $P$ value $<0.001$. Recurrence occurred in $(14.3 \%)$ of cases in urographin group and in (33.3\%) of cases in conservative group

Conclusion: The period of hospital admission is significantly shorter with the use of oral water soluble contrast (urographin) with fewer incidences of recurrent attacks but it doesn't decrease the incidence of shifting to surgery. The use of urographin is safe with no significant harmful side effects observed.

Key words: Oral urographin, early adhesive intestinal obstruction.

\section{Introduction}

Adhesive bowel obstruction is considered as an important cause of postoperative morbidity in pediatrics. Appendectomy, performing stoma and its closure, fundoplication, and operated neonatal intestinal obstruction are the most common procedures causing adhesive small bowel obstruction, Its incidence in the literature ranges from 2 to $30 \%$ and is more in neonates, mostly occurring within 2 years after surgical intervention. ${ }^{1,2}$ Intestinal injury is reported up to $30 \%$ of patients undergoing surgery for adhesive bowel obstruction with increased postoperative morbidity, so conservative measures of managing adhesive intestinal obstruction have generated considerable interest, among which; water soluble contrast is proved to be effective in adults. $^{3-5}$

The role of using water soluble contrast in adhesive bowel obstruction, has been evaluated in the last few years with regard to diagnostic and therapeutic role, its osmolarity approximately 6 times more than the extracellular fluid osmolarity, promotes shifting of fluids to the intestinal lumen and increases the pressure gradient at obstructive sites. Furthermore, because water soluble contrast can dilute the bowel contents, it facilitates the passage of intestinal contents and decreases edema in the bowel wall. ${ }^{6,7}$

The aim of this study is to evaluate the therapeutic role of water soluble contrast (urographin) in treatment of adhesive intestinal obstruction in pediatric population.

\section{Patients and methods}

After acceptance of the scientific and ethical committee of our institution, this prospective comparative study was conducted in pediatric surgery unit, Sohag university hospital and Qena university hospital over 12 months period between December 2016 to December 2017 on all pediatric patients who presented by picture of early adhesive intestinal obstruction. Patients who improved in the first 24 hours and patients with clinical, laboratory, or radiological evidence of strangulation were excluded.

Clinical evaluation (mainly to exclude other possible causes of intestinal obstruction and to exclude any clinical evidence of strangulation), routine laboratory investigations, and Imaging studies (plain $\mathrm{x}$ - ray erect and abdominal ultrasound) were done in all cases (Figure 1). 


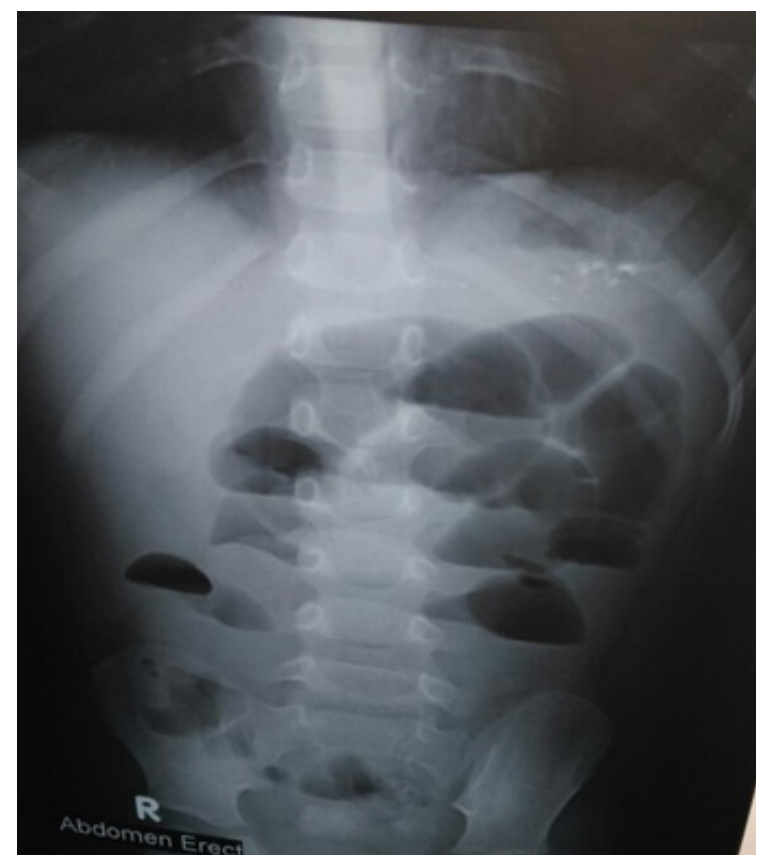

Fig 1: Plain $X$ ray show multiple fluid levels.

All patients were given conservative treatment for 24 hours (nothing per mouth, insertion of nasogastric tube, and intravenous fluids), patients with failure of improvement after 24 hours were divided to 2 groups in the first group we continued the same conservative measures for 48 hours, in the second group we used single oral dose of urographin \{dosage was age- dependent, with children age 8 years or older receiving $200 \mathrm{~mL}$, and those under age 8 years receiving $100 \mathrm{~mL}$.\} (Figure 2).

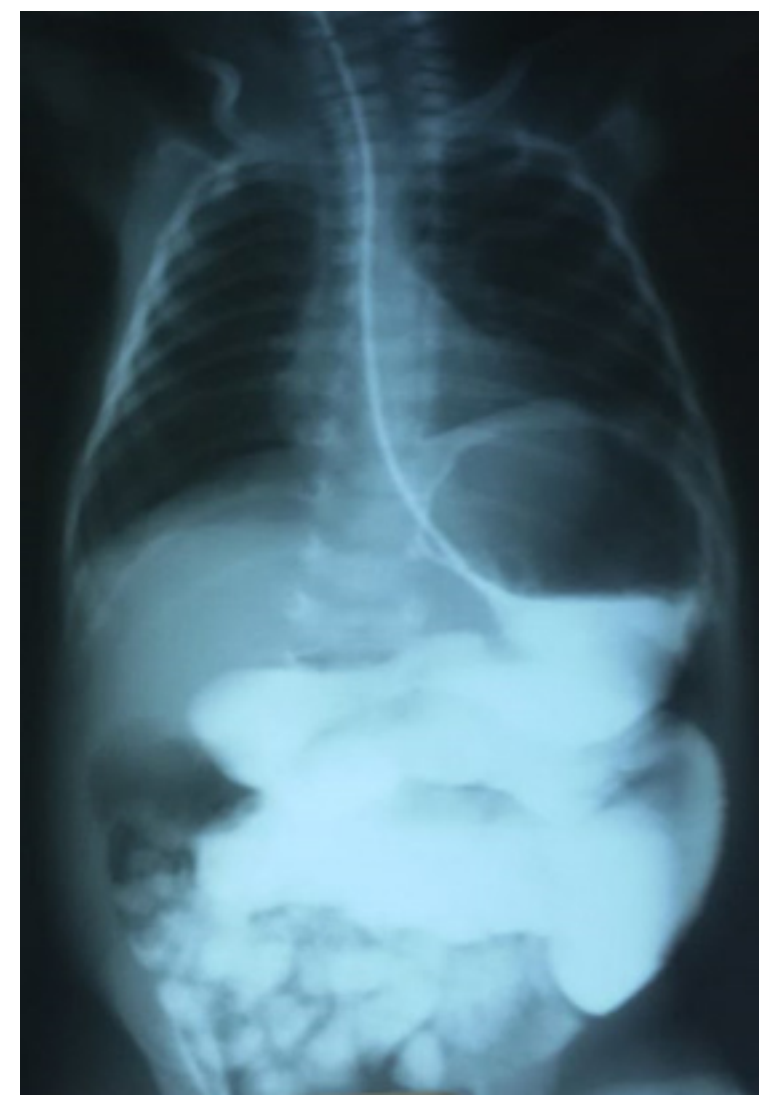

Fig 2: Oral dose of gastrografin.
Follow up of all patients was done clinically and radiologically for another $\mathbf{4 8}$ hours. Surgical intervention (operative adhesiolysis) was done with clinical and radiological failure of conservative treatment after 72 hours (Figure $\mathbf{3}$ ) or when signs of strangulation appeared during period of conservation. Patients (without surgical intervention) were discharged 24 hours of clinical and radiological improvement, and follow up was done for 12 months to evaluate incidence of recurrent episodes. Other data as: demographic data, type of first surgery, period of conservation, results, complications, and outcome all were collected and statistically analyzed.

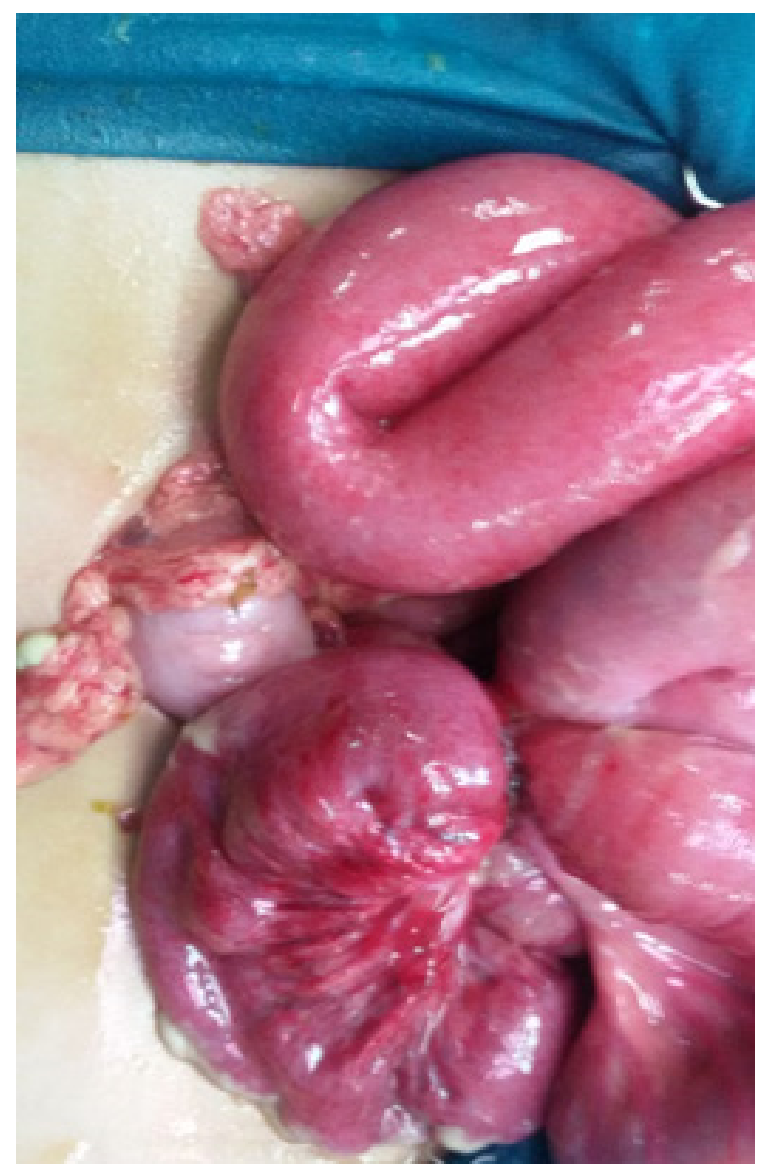

Fig 3: Intra-operative adhesion.

\section{Results}

In the period from December 2016 to December 2017, 35 patients were admitted with picture of early adhesive intestinal obstruction, after 24 hours of traditional conservative measures: (nothing per mouth, nasogastric tube, and intravenous fluids) patients were divided into 2 groups (Table 1), the first group included 21 patients in which we continued the same regimen of conservation and the second group included 14 patients in which single oral dose of urographin (dosage was age- dependent, with children age 8 years or older receiving $200 \mathrm{~mL}$, and those under age 8 years receiving $100 \mathrm{~mL}$.) was given. With follow up of patients for 48 hours, any patient with any evidence of strangulation was excluded(leukocytosis, tachycardia, rebound tenderness\& fever) (Table 2). 
Table 1: Comparison between the studied groups regarding age and sex

\begin{tabular}{|c|c|c|c|}
\hline Characteristics & $\begin{array}{c}\text { Conservative } \\
(\mathrm{N}=21)\end{array}$ & $\begin{array}{l}\text { Oral gastrografin } \\
(N=14)\end{array}$ & P-value \\
\hline \multicolumn{4}{|l|}{ Age (months) } \\
\hline Mean \pm S.D. & $74.43 \pm 43.4$ & $62.86 \pm 44.64$ & \multirow[t]{2}{*}{$0.359 *$} \\
\hline Median (Range) & $69(11-170)$ & $46.5(9-148)$ & \\
\hline \multicolumn{4}{|l|}{ Sex } \\
\hline Female & $12(57.1 \%)$ & $4(28.6 \%)$ & \multirow[t]{2}{*}{0.096} \\
\hline Male & $9(42.9 \%)$ & $10(71.4 \%)$ & \\
\hline
\end{tabular}

Table 2: Comparison between the studied groups regarding pulse and WBCs at presentation

\begin{tabular}{lccc}
\hline Characteristics & $\begin{array}{c}\text { Conservative } \\
(\mathbf{N}=\mathbf{2 1})\end{array}$ & $\begin{array}{c}\text { Oral gastrografin } \\
(\mathbf{N}=\mathbf{1 4})\end{array}$ & P-value \\
\hline Pulse at presentation & $79.81 \pm 3.99$ & $81.64 \pm 4.83$ & 0.23 \\
$\quad$ Mean \pm S.D. & $80(70-86)$ & $82(72-88)$ & 0.949 \\
Median (Range) & & $8.29 \pm 2.49$ & $8(4-12)$ \\
WBCs $\left(\mathbf{1 0}^{\mathbf{3}} / \boldsymbol{\mu L}\right)$ at presentation & $8.24 \pm 1.89$ & & \\
Mean \pm S.D. & $8(5-11)$ &
\end{tabular}

In our study operated acute appendicitis was the commonest cause of adhesive intestinal obstruction (Table 3). Surgical intervention was indicated in 6 cases in the conservative group and in 4 cases in gastrografin group (Table 4 ). Serosal tears occurred in 2 patients during surgery, and full thickness injury occurred in one patient in whom primary repair was done by single layer mucosa excluding repair with smooth postoperative course.

Table 3: Comparison between groups regarding previous surgery

\begin{tabular}{|c|c|c|c|}
\hline Previous surgery & $\begin{array}{c}\text { Conservative } \\
(\mathbf{N}=\mathbf{2 1})\end{array}$ & $\begin{array}{l}\text { Oral gastrografin } \\
\qquad(\mathrm{N}=14)\end{array}$ & P-value \\
\hline Acute appendicitis & $4(19 \%)$ & $1(7.1 \%)$ & \multirow{14}{*}{0.219} \\
\hline Complicated Meckel's & $1(4.8 \%)$ & $0(0.0 \%)$ & \\
\hline Abdominal wall defects & $2(9.5 \%)$ & $2(14.3 \%)$ & \\
\hline Intestinal atresia & $1(4.8 \%)$ & $2(14.3 \%)$ & \\
\hline Intestinal perforation(trauma) & $0(0.0 \%)$ & $2(14.3 \%)$ & \\
\hline Mesenteric cyst & $1(4.8 \%)$ & $0(0.0 \%)$ & \\
\hline Mesenteric injury(trauma) & $0(0.0 \%)$ & $1(7.1 \%)$ & \\
\hline Midgut volvulus & $0(0.0 \%)$ & $2(14.3 \%)$ & \\
\hline NEC with colostomy & $1(4.8 \%)$ & $0(0.0 \%)$ & \\
\hline Intussusception & $4(19 \%)$ & $1(7.1 \%)$ & \\
\hline Neglected obstructed cong hernia with resection & $2(9.5 \%)$ & $1(7.1 \%)$ & \\
\hline Infected ventriculoperitoneal shunt & $1(4.8 \%)$ & $0(0.0 \%)$ & \\
\hline Perforated appendix & $3(14.3 \%)$ & $1(7.1 \%)$ & \\
\hline Splenectomy (trauma) & $1(4.8 \%)$ & $1(7.1 \%)$ & \\
\hline
\end{tabular}


Table 4: Comparison between the studied groups regarding recurrence, complications and management related data

\begin{tabular}{lccc}
\hline Characteristics & $\begin{array}{c}\text { Conservative } \\
(\mathbf{N = ~ 2 1})\end{array}$ & $\begin{array}{c}\text { Oral gastrografin } \\
(\mathbf{N = 1 4 )}\end{array}$ & P-value \\
\hline Shifting to surgery & $15(71.4 \%)$ & $10(71.4 \%)$ & 1 \\
$\quad$ No & $6(28.6 \%)$ & $4(28.6 \%)$ & \\
$\quad$ Yes & $14(66.7 \%)$ & $12(85.7 \%)$ & 0.262 \\
Recurrence within 12 months & $7(33.3 \%)$ & $2(14.3 \%)$ & \\
No & & & \\
Yes & $6.24 \pm 0.89$ & $3.36 \pm 0.49$ & $<0.001^{* *}$ \\
Hospital stay (days) & $6(4-8)$ & $3(3-8)$ & \\
$\quad$ Mean \pm S.D. & & & \\
Median (Range) &
\end{tabular}

\section{Discussion}

Adhesive small bowel obstruction in pediatrics is considered one of the most important elements of postoperative morbidity with increased risk of morbidity including intestinal injury with the use of operative adhesiolysis as main line of treatment, and so searching for effective non operative treatment may lead to less morbidity, less hospital stay, and so will be cost saving. ${ }^{8}$

The use of oral water-soluble contrast as a diagnostic and therapeutic tool for adhesive small bowel obstruction in the adult population proved to beneficial in many medical reports As regards diagnosis, oral contrast transit from stomach to large intestine is reported to predict spontaneous resolution of adhesive small bowel obstruction with $96 \%$ sensitivity and $98 \%$ specificity. Moreover many authors reported fewer incidences of surgical intervention, and shorter time for conservative treatment and hospital stay with the use of oral water soluble contrast. ${ }^{9}$

As regards the etiology of adhesive bowel obstruction in different age groups many authors showed that appendectomy and colorectal procedures are the most common procedures, , in our study in pediatric patients acute appendicitis was the commonest followed by operated intussusception. ${ }^{10}$

The duration of conservation is controversial between authors but almost all authors recommend surgical intervention if conservation failed after 4872 hours. In our institution we shifted to surgery 72 hours after conservative treatment provided there was no any of the manifestations of strangulation during the close observation period. ${ }^{11,12}$ gastrografin significantly increased the incidence of resolution of obstruction, decreased the hospital stay duration, and reduced the need for surgical intervention to $10 \%$. Another similar study concluded that urografin is a safe and reliable water-soluble contrast medium, that can be used to differentiate partial intestinal obstruction from complete intestinal obstruction, and that early oral intake was found to be a major advantage of urografin use. However, Feigin et al and Fevang et al concluded that there is no change of the operative rate, time of resolution of obstruction, and hospital stay. ${ }^{11,13-15}$

In this study the duration of hospitalization significantly decreased with the use of gastrografin, the recurrence attacks (during one year period of follow up) decreased with its use but the incidence of shifting to surgery did not change.

This study was limited by the small number of patients, also due to different causes of adhesive intestinal obstruction, we did not take into account direct correlation of results dependent on primary cause, in the other hand long follow up is needed for evaluation of rate of re-exploration in both groups, we also need assessment of cost and utilization of hospital resources in both group, lastly we need to evaluate efficacy of giving another dose of oral contrast in cases not improving with first one.

\section{Conclusion}

The period of hospital admission is significantly shorter with the use of oral urografin with fewer incidences of recurrent attacks but it doesn't decrease the incidence of shifting to surgery, the use of gastrografin is safe with no significant harmful side effects observed. 


\section{References}

1. Eeson GA, Wales P, Murphy JJ: Adhesive small bowel obstruction in children: Should we still operate? J Pediatr Surg. 2010; 45: 969-974.

2. Gingalewski CA: Other causes of intestinal obstruction. In: Grosfeld J, O'Neill J Jr, Fonkalsrud E, Coran AG, Caldamone AA, editors. Pediatric Surgery. $6^{\text {th }}$ ed. Philadelphia, PA: Elsevier Science. 2006: 1358-1368.

3. Van der Krabben AA, Dijkstra FR, Nieuwenhuijzen $M$, et al: Morbidity and mortality of inadvertent enterotomy during adhesiolysis. Br $\boldsymbol{J}$ Surg. 2000; 87: 467-471.

4. Di Saverio S, Catena F, Ansaloni L, Gavioli M, Valentino M, Pinna AD: Water-soluble contrast medium (gastrografin ) value in adhesive small intestine obstruction (ASIO): A prospective, randomized, controlled, clinical trial. World $\boldsymbol{J}$ Surg. 2008; 2304-32;2293.

5. Kapoor S, Jain G, Sewkani A, Sharma S, Patel $\mathrm{K}$, Varshney S: Prospective evaluation of oral gastrografin in postoperative small bowel obstruction. J Surg Res. 2006; 131: 256-260.

6. Choi HK, Chu KW, Law WL: Therapeutic value of Gastrografin in adhesive small bowel obstruction after unsuccessful conservative treatment: A prospective randomized trial. Ann Surg. 2002; 236: $1-6$.

7. Biondo S, Pares D, Mora L, Marti Rague J, Kreisler E, Jaurrieta E: Randomized clinical study of gastrografin administration in patients with adhesive small bowel obstruction. $\boldsymbol{B r} \boldsymbol{J}$ Surg. 2003; 90: 542-546.

8. Zielinski M, Haddad N, Choudhry A, et al: Multi-institutional, prospective, observational study comparing the gastrografin challenge versus standard treatment in adhesive small bowel obstruction. J Trauma Acute Care Surg. 2017; 83: 47-54.

9. Linden $A F$, Raiji MT, Kohler JE, et al: Evaluation of a water-soluble contrast protocol for nonoperative management of pediatric adhesive small bowel obstruction. J Pediatr Surg. 2019; 54(1): 184-188.

10. Cox MR, Gunn IF, Eastman MC, et al: The operative aetiology and types of adhesions causing small bowel obstruction. Aust $N Z J$ Surg. 1993; 63: 848-852. [PubMed].

11. Assalia A, Schein M, Kopelman D, et al: Therapeutic effect of oral gastrografin in adhesive, partial small-bowel obstruction: A prospective randomized trial. Surgery. 1994; 115: 433-437. [PubMed].

12. Sosa J, Gardner B. Management of patients diagnosed as acute intestinal obstruction secondary to adhesions. Am Surg. 1993; 59: 125-128. [PubMed].

13. Feigin $E$, Seror $D$, Szold $A$, Carmon $M$, Allweis TM, Nissan A, Gross E, Vromen A, Freund R: Water-soluble contrast material has no therapeutic effect on postoperative smallbowel obstruction: Results of a prospective, randomized clinical trial. Am J Surg. 1996; 171: 227-229.

14. Shyr-Chyr C, Jen C, Huang L, et al: Oral urografin in postoperative small bowel obstruction. World Journal of Surgery. 1999; 23(10): 1051-1054.

15. Fevang BT, Jensen D, Fevang J, et al: Upper gastrointestinal contrast study in the management of small bowel obstruction-A prospective randomized study. Eur J Surg. 2000; 166: 39-43. [PubMed]. 